\title{
EVALUATION OF MINERALIZED PLASMATIC MATRIX AS A GRAFTING MATERIAL AROUND IMMEDIATE DENTAL IMPLANT IN MANDIBULAR POSTERIOR TEETH
}

\author{
Ahmed Abed El-Sayd Mansour ${ }^{1 *}$, Mohamed Abd Elsalam Khalil ${ }^{2}$ and Mohammad Abdel-Hamied Shuman ${ }^{3}$
}

\begin{abstract}
Objective: the present study was performed to evaluate Mineralized Plasmatic Matrix (MPM) as a grafting material around immediate dental implant in mandibular posterior teeth. Subjects and Methods: Eight patients were selected for immediate dental implant in mandibular posterior teeth and use MPM as a grafting material around the implant. After surgery each patient were evaluated clinically at the following interval immediate, one week, two weeks, and three months postoperatively for:: Presence of infection, Edema, Graft exposure and/or loss, Soft tissue dehiscence, Pain, Nerve injury and Implant stability, and radiographically immediate and three months postoperatively interval by $\mathrm{CBCT}$ for: Bone density around the dental implant and Horizontal bone loss around the dental implant. Results: All patients hadn't any infection, nerve injury, Graft exposure and /or loss, soft tissue dehiscence and facial edema throughout the study till three months. Osstel at 3 months $(81.0 \pm 7.68)$ was higher than immediately

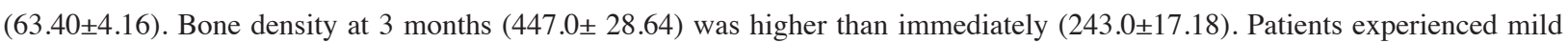
to moderate pain after surgery, at the surgical sites which decrease gradually during four days postoperatively then disappeared completely at the $7^{\text {th }}$ day. Conclusion: The use of MPM with immediate implant reduces postoperative infection, edema, pain, graft exposure and/or loss and soft tissue dehiscence. Use of MPM with immediate implant enhances the implant stability, improves bone density around it and decrease marginal bone loss around the immediate implant.
\end{abstract}

KEY WORDS: Mineralized Plasmatic Matrix; immediate implant; bone density; marginal bone loss; implant stability.

\section{INTRODUCTION}

Dental implants are alloplastic materials embedded in the maxilla and/or mandible for the management of tooth loss and to aid replacement of lost orofacial structures as a result of trauma, neoplasia and congenital defects. Implant supported dental restorations have become a major option in the treatment of edentulous alveolar ridge for the past several decades. To achieve optimum treatment outcome with dental implants, sufficient bone should be available to support and stabilize them ${ }^{(1)}$. Bone volume is one of the key factors to be considered when evaluating implant placement. When the bone volume is insufficient, implant placement could be conditioned by the necessity of preforming bone grafting procedures to compensate bone loss ${ }^{(2)}$.

1. Masters Candidate, Department of Oral and Maxillofacial Surgery, Faculty of Dental Medicine, Al-Azhar University, Boys, Cairo

2. Professor, Department of Oral and Maxillofacial Surgery, Faculty of Dental Medicine, Al-Azhar University, Boys, Cairo

3. Assistant Professor, Department of Oral and Maxillofacial Surgery, Faculty of Dental Medicine, Al-Azhar University, Boys, Assuit.

-Corresponding author: aam.mansour@yahoo.com

DOI: 10.21608 /ajdsm.2020.38219.1088 
Alveolar bone defects occur due to periodontitis, trauma, tumors or resorption following tooth extraction. Extensive loss of alveolar bone presents a complex challenge for reconstruction. Soft and hard tissue defects create an anatomically less favorable context for ideal implant placement ${ }^{6}$. Reconstruction of the alveolar bone through a variety of regenerative surgical procedures had become predictable ${ }^{(3)}$. Autogenous, allogenic and tissue engineered bone grafts are successfully used. The success rates of them have become better in the last decades ${ }^{(4)}$. Various grafting procedures can be used with different bone substitute. The introduction of protein therapy in regenerative procedures could overcome the use of rigid membranes in certain cases making grafting procedures easier ${ }^{(2,4)}$. In order to accelerate healing of bone graft over the bony defect, numerous techniques utilizing platelet and fibrinogen concentrations have been introduced recently ${ }^{(1)}$.

Platelets are known to contain high quantities of growth factors, such as transforming growth factors $\beta-1$ (TGFß-1), platelet-derived growth factor (PDGF), epithelial growth factor (EGF), insulin growth factor-I (IFG-I) and vascular endothelial growth factors (VEGF) which may have a role in stimulating cell proliferation and up regulating angiogenesis ${ }^{(5)}$. Mineralized plasmatic matrix (MPM) is used as one of the most recent grafting materials. It is an autologous blood product highly concentrated in platelets and fibrin combined with bone substitute $^{(4)}$. The fibrin can become bound to bone particles and the filling material is easy to shape ${ }^{(6)}$.

\section{SUBJECTS AND METHODS}

The present study was performed to evaluate Mineralized Plasmatic Matrix (MPM) as a grafting material around immediate dental implant in mandibular posterior teeth. Patients were selected from those attending outpatient clinic of oral and maxillofacial surgery department at faculty of dental medicine, Al-Azhar University, Boys, Cairo.

\section{Inclusion criteria:}

Presence of a non-restorable mandibular posterior tooth indicated for extraction with good oral hygiene.

\section{Exclusion criteria:}

Limited mental capacity or language skills or suffering from a known psychological disorder. Patient on chemotherapy, radiotherapy or finished the therapy from less than 6 months. Acute periapical pathosis in the tooth under study or in the neighboring teeth which could affect surgery. Infections or recent surgical procedures within 1 month of baseline visit known to affect oral status or contraindicate surgical treatment. Pregnancy or lactating period. Heavy smoker's patients. Patient who was receiving or had been exposed to bisphosphonate therapy.

\section{Clinical parameters:}

All patients were evaluated clinically for: Presence of infection: Patients were assessed objectively and subjectively for cardinal signs of infection such as: redness, hotness, pain, swelling, purulent discharge, odour ${ }^{(7)}$. Edema: Facial measurements were obtained using a measuring tape while the patient was sitting upright and the mandible was in the physiologic rest position. Five points on the face were used: most posterior point at midline on tragus (A), lateral canthus of eye (B), most lateral point on corner of mouth (C), soft tissue pogonion, which is the most prominent point at midline on chin (D), and most inferior point on the angle of the mandible (E) . The measurements for these three lines, (A to $\mathrm{C}$ ), (B to $\mathrm{E}$ ), and (A to $\mathrm{D}$ ), were recorded three times, then the average was taken ${ }^{(8)}$. Graft exposure and/or loss, and Soft tissue dehiscence: by visual inspection. Nerve injury: Postoperative assessment was done for paresthesia or anesthesia by questioning about tongue, chin, and lip sensibility and performing neurosensory tests like light touch. These parameters were evaluated immediate postoperative and at one week, one month, and three months intervals. Pain: was assessed six hours, two days, four 
days and seven days following surgery using the visual analog scale (VAS) ${ }^{(9)}$. Implant stability: Stability of the implant was monitored via the Resonance Frequency Analysis (RFA) by Osstell device at immediate (intra-operative) and three months postoperatively only ${ }^{(10)}$.

\section{Radiographic parameters:}

Bone density: The buccal and lingual peri-implant bone density (relative) changes were assessed from the coronal sagittal cuts while the mesial and distal changes were assessed from the panoramic one using the density measurement tool to determine the region of interest (ROI) then the bone density in Hounsfield unit (HU) was automatically calculated by the software. Relative bone density was measured then the average of the bone density of the four surfaces was calculated ${ }^{(11)}$. Horizontal bone loss around the dental implant: Assessing bone dimensional changes was done using the linear measurement tool supplied by CBCT software (OnDemand3D software) by measuring the distance between the platform and the buccal and lingual cortical plates on the cross-sectional view and from the mesial and distal crestal bone margins and implant platform in the panoramic view of CBCT radiograph and taking the average of these four readings (12). These parameters were evaluated immediate postoperative and at three months intervals.

\section{Surgical procedure (stage I):}

Preoperative antibiotic were administered orally 1 hour befor procedure. All patients were instructed to rinse with $0.12 \%$ chlorhexidine gluconate mouth wash immediately before operation for 30 seconds. All procedures were performed under local anesthesia using articane Inferior alveolar nerve block anesthesia followed by buccal infiltration anesthesia was administered. Following the performance of local anesthesia, Removal of the non-restorable mandibular molar was performed by atraumatic surgical extraction. For all teeth extraction, a priotome was used to severe surrounding periodontal ligament attachment after tooth sectioning. Then the roots were finally delivered using extraction forceps. Extreme care to preserve integrity of buccal and lingual bone plates. For all cases following tooth extraction a proper debridement of extraction sockets using bone curettes followed by copious irrigation.

\section{Preparation of mineralized plasmatic matrix (MPM):}

Ten millimeters of patient's venous blood were obtained by direct puncture to a vein, most often located in the antecubital area of the arm or the back (top) of the hand, using a vascular access device into the tubes. Two tubes were collected. The venous blood was placed into the centrifugation machine to separate the red blood cells from the platelets for $8 \mathrm{~min}$ at $2700 \mathrm{RPM}$. The yellow plasma liquid on the top of the tube separated from the red blood cells in its bottom. The yellow part was collected using a syringe and added to a cup that contains the bone grafting material, followed by mixing for few seconds then MPM was obtained. PRF gel as intermediate layer (buffy coat) was squeezed to obtain membrane-like platelet rich fibrin (PRF) and used for clinical applications.

\section{Implant placement procedure:}

After that a round shaped bur no. 8 was used to create a dimple through the area of proposed implant site. Drilling was done with an up and down motion following the recommended sequence of the manufacturer instructions under copious external irrigation. Pilot drill was used to create a pilot hole to the predetermined length of the implant. Parallelism with adjacent natural teeth was checked by intraoperative periapical radiograph with the paralleling pin in place. Drills were used according to predetermined diameter of implant in sequence to the full length. Implant was installed in the interseptal bone after sequential drilling, after that implant primary stability was measured using Osstell device ${ }^{(10)}$. The jumping gap around the implant in the extraction socket was completely packed using MPM followed by coverage using PRF membrane. The membrane was stabilized using $\mathrm{X}$-shaped suture. 


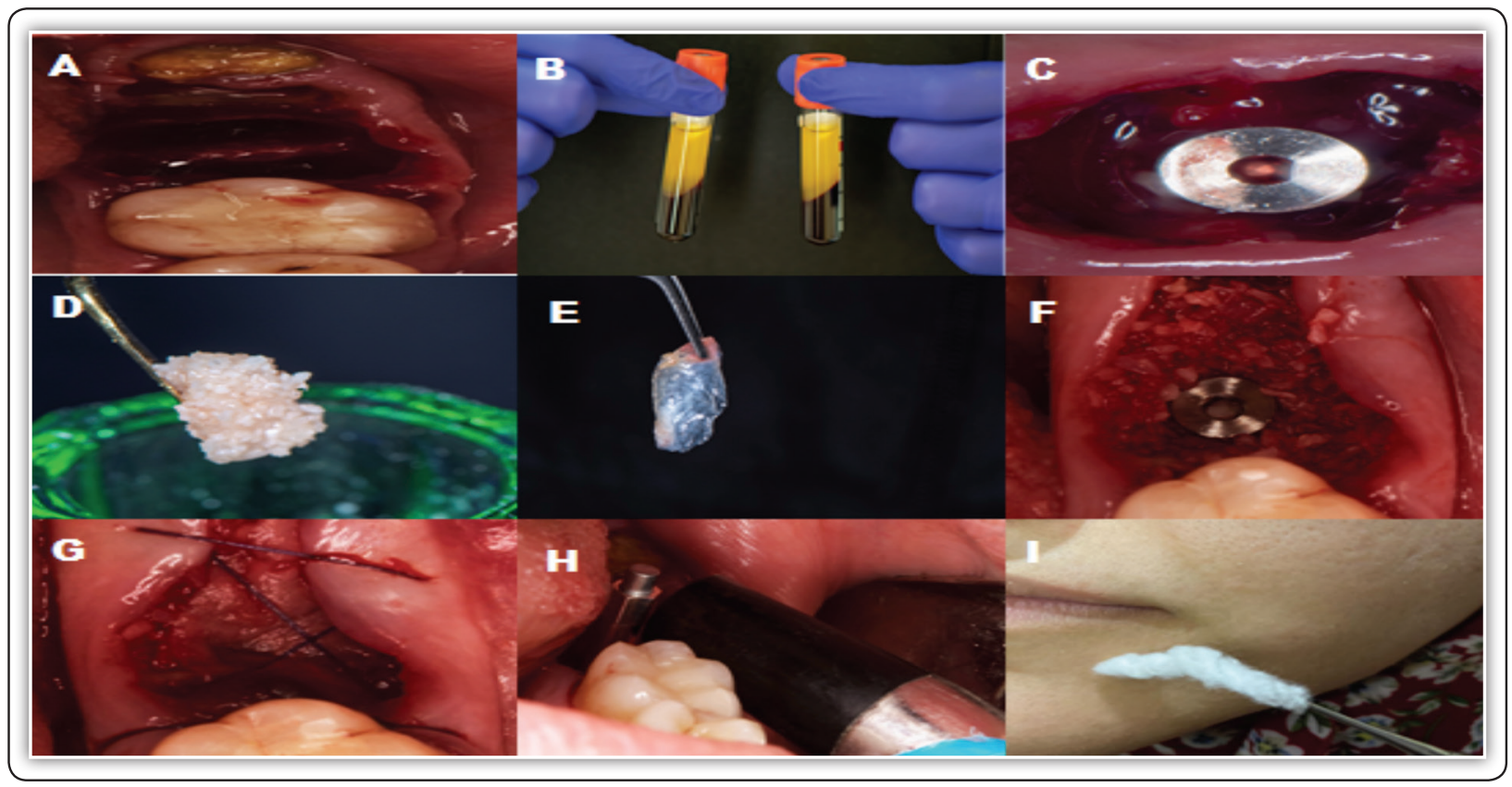

FIG (1):A; Empty socket after tooth extraction, B; Blood sample centrifuged, C; Socket after implant insertion, D; MPM, E; PRF membrane, F; Defects around implant filled with MPM, G; Socket covered by PRF membrane and suturing, H; Measuring immediate implant stability, and I; Light touch assessment

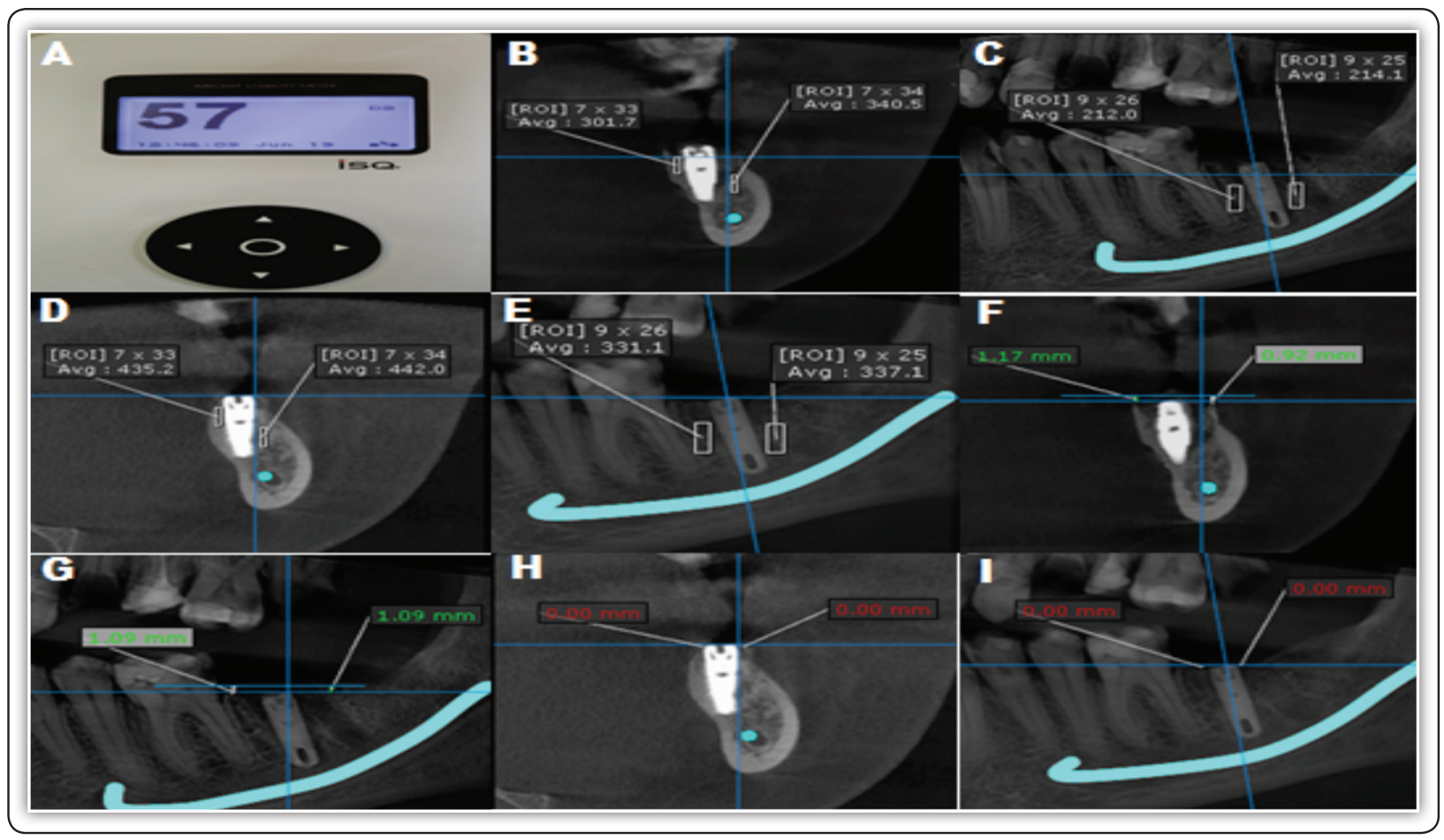

FIG (2):A; Osstell device reading, B\&C; Evaluation of relative bone density immediately, D\&E; Evaluation of relative bone density 3 months postoperatively, F\&G; Evaluation of horizontal bone loss immediately, and H\&I; Evaluation of horizontal bone loss 3 months postoperatively. 


\section{Surgical procedure (stage II):}

Surgical stage II was carried on 3 months after the first surgery. The cover screw was probed (if covered by soft tissue). The cover screw was removed and the area flushed with normal saline. Stability was measured using Ostell device. Standard oral hygiene measures were instructed for the patient as before in the stage I surgery.

\section{RESULTS}

The present study was to evaluate Mineralized Plasmatic Matrix as a grafting material around immediate dental implant in mandibular posterior teeth. In the present study, five patients ranged in age between $30.0-56.0$ years with a mean age of 41.25 \pm 8.65 years. Patients were 3 males and 5 females. All patients hadn't any signs of infection such as: redness, hotness, pain, swilling, purulent discharge or odour. And so, no patient had any infection, nerve injury, facial edema, soft tissue dehiscence and graft exposure and/or loss throughout the study till three months (Table 1\&2). Pain: Patients experienced mild to moderate pain after surgery, at the surgical sites which decrease gradually during four days postoperatively then disappeared completely at the $7^{\text {th }}$ day. And so on there was a statistically significant difference in mean Pain after one week (Table 3). Implant stability there was increase in the mean of implant stability after three months postoperatively $(67.00 \pm 7.27)$ where it was $(55.13 \pm 7.74)$ immediately. This change was a statistically significant. Bone density there was increase in the mean of bone density after three months $(400.25 \pm 59.87)$ where it was $(230.25 \pm 25.36)$ immediately. This change was a statistically significant. Marginal bone loss there was slightly increase in the mean of marginal bone loss after three months $(0.03 \pm 0.06)$ where it was $(0.0)$ immediately. However, This change was a statistically non-significant (Table 4).

TABLE (1): Comparison between the different time periods according to infection, nerve injury, graft exposure and/or loss, and soft tissue dehiscence $(n=8)$

\begin{tabular}{|c|c|c|c|c|c|c|c|c|c|}
\hline & \multicolumn{2}{|c|}{ Immediate } & \multicolumn{2}{|c|}{ One week } & \multicolumn{2}{|c|}{ Two weeks } & \multicolumn{2}{|c|}{ Three months } & \multirow{2}{*}{$\mathbf{P}$} \\
\hline & No. & $\%$ & No. & $\%$ & No. & $\%$ & No. & $\%$ & \\
\hline \multicolumn{10}{|l|}{ Infection } \\
\hline Negative & 8 & 100.0 & 8 & 100.0 & 8 & 100.0 & 8 & 100.0 & \multirow{2}{*}{-} \\
\hline Positive & 0 & 0.0 & 0 & 0.0 & 0 & 0.0 & 0 & 0.0 & \\
\hline \multicolumn{10}{|c|}{ Nerve injury } \\
\hline Negative & 8 & 100.0 & 8 & 100.0 & 8 & 100.0 & 8 & 100.0 & \multirow{2}{*}{-} \\
\hline Positive & 0 & 0.0 & 0 & 0.0 & 0 & 0.0 & 0 & 0.0 & \\
\hline \multicolumn{10}{|c|}{ Graft exposure and /or loss } \\
\hline Negative & 8 & 100.0 & 8 & 100.0 & 8 & 100.0 & 8 & 100.0 & \multirow{2}{*}{ - } \\
\hline Positive & 0 & 0.0 & 0 & 0.0 & 0 & 0.0 & 0 & 0.0 & \\
\hline \multicolumn{10}{|c|}{ Soft tissue dehiscence } \\
\hline Negative & 8 & 100.0 & 8 & 100.0 & 8 & 100.0 & 8 & 100.0 & \multirow{2}{*}{ - } \\
\hline Positive & 0 & 0.0 & 0 & 0.0 & 0 & 0.0 & 0 & 0.0 & \\
\hline
\end{tabular}


TABLE (2): Comparison between the different time periods according facial edema ( $\mathrm{n}=8)$

\begin{tabular}{|c|c|c|c|c|c|c|c|c|c|c|}
\hline & \multicolumn{2}{|c|}{ Immediate } & \multicolumn{2}{c|}{ One week } & \multicolumn{2}{c|}{ One month } & \multicolumn{2}{c|}{ Three months } & \multirow{2}{*}{$\mathrm{F}$} & \multirow{2}{*}{$\mathrm{p}$} \\
\cline { 2 - 12 } & Mean & $\pm \mathrm{SD}$ & Mean & $\pm \mathrm{SD}$ & Mean & $\pm \mathrm{SD}$ & Mean & $\pm \mathrm{SD}$ & & \\
\hline Facial edema & 12.49 & 1.03 & 12.55 & 1.06 & 12.49 & 1.03 & 12.49 & 1.03 & 1.093 & 0.364 \\
\hline $\mathrm{p}_{\text {Imm. }}$ & & & \multicolumn{2}{|c|}{1.000} & \multicolumn{2}{|c|}{1.000} & \multicolumn{2}{|c|}{1.000} & & \\
\hline$\%$ of Change & & & $\uparrow 0.22$ & 0.35 & $\uparrow 0.41$ & 0.69 & $\uparrow 0.05$ & 0.11 & & \\
\hline
\end{tabular}

TABLE (3): Comparison between the different time periods according pain $(n=8)$

\begin{tabular}{|c|c|c|c|c|c|c|c|c|c|c|}
\hline & \multicolumn{2}{|c|}{6 Hours } & \multicolumn{2}{c|}{ Two days } & \multicolumn{2}{c|}{ Four days } & \multicolumn{2}{c|}{ One week } & \multirow{2}{*}{ Fr } & \multirow{2}{*}{$\mathrm{p}$} \\
& Mean & $\pm \mathrm{SD}$ & Mean & $\pm \mathrm{SD}$ & Mean & $\pm \mathrm{SD}$ & Mean & $\pm \mathrm{SD}$ & & \\
\hline Pain & 3.75 & 0.89 & 3.63 & 0.92 & 2.25 & 0.89 & 0.0 & 0.0 & $19.95^{*}$ & \multirow{2}{*}{$0.0017^{*}$} \\
\hline $\mathrm{p}_{\text {procedure }}$ & & & \multicolumn{2}{|c|}{$0.049^{*}$} & \multicolumn{2}{|c|}{$0.010^{*}$} & \multicolumn{2}{|c|}{$0.005^{*}$} & & \\
\hline$\%$ of Change & & & 90.30 & 12.38 & 94.00 & 0.0 & 100.0 & 0.0 & & \\
\hline
\end{tabular}

TABLE (4): Comparison between immediate and three months according to implant stability $(\mathrm{n}=8)$

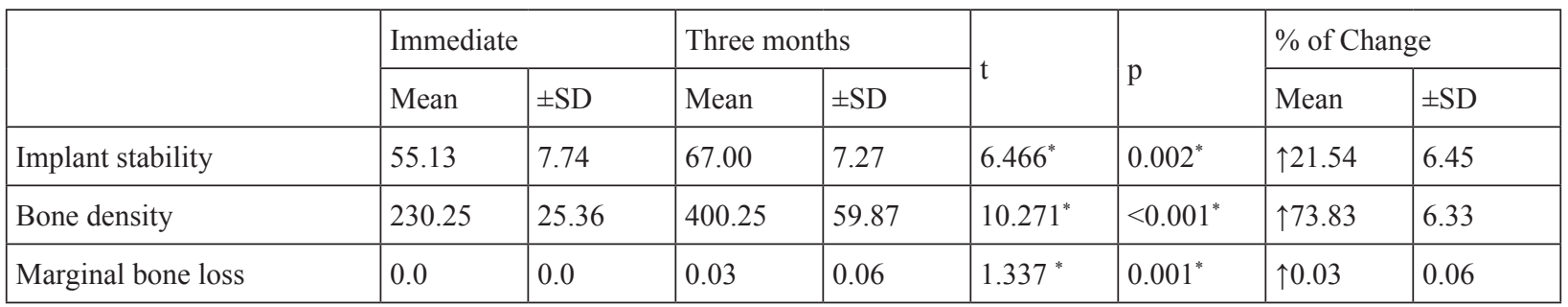

\section{DISCUSSION}

We found that Mineralized Plasmatic Matrix can be used as a grafting material around immediate dental implant in mandibular posterior teeth. As we found that MPM reduce postoperative infection, edeama, pain, Graft exposure and/or loss and soft tissue dehiscence. Also MPM improve stability of immediate implant and enhance the bone density around it.

In our result no one case of infection was found. Infection may follow dental implant therapy in the first few postoperative days; it is caused by bacterial contamination during surgery. Beside the strict adherence to the surgical principles of asepsis, this may be due to platelets play a role in host defense mechanisms at the wound site, by delivering signaling peptides which attract macrophage cell, platelet concentrates may contain small amounts of leukocytes that synthesize interleukins involved in the non-specific immune reaction and Antimicrobial activity of platelet concentrates against several bacterial species involved in oral infections has also been reported ${ }^{(13)}$.

Regarding postoperative infection, our result is in agreement with Medikeri et al. They found combined use of growth factors with pre- and postoperative broadspectrum antibiotics over a short time resulted in a higher implant survival rate in infected site. Hoaglin et al found that we can Prevent localized osteitis in mandibular third molar sites using platelet rich fibrin ${ }^{(14,15)}$. 
Results of the present study have a statisticvally non-significant difference in mean of facial edema during the postoperative three months, indicate that using MPM has significant effect on the postoperative edema following dental implant surgery. That is due to MPM deliver the growth factors in high concentration to the surgical site. These growth factors accelerate post-surgical healing and decrease postoperative inflammation (6). Regarding postoperative edema, our result is in agreement with Muñoz et al. using PRF in Combination with traditional bone grafts potentially accelerates wound healing and reduces post-surgical inflammation ${ }^{(16)}$. Similar result was also reported by Ozgul et $\mathrm{al}^{(17)}$.On the other hand, it is in controversy with Torul D who found that PRF seem to have no positive effects edema after third molar surgery ${ }^{(18)}$.

Results of the present study have a statistically significant difference in mean of pain during the postoperative seven days; indicate that using MPM has significant effect on the postoperative pain following dental implant surgery. That is due to MPM deliver the growth factors in high concentration to the surgical site. These growth factors accelerate post-surgical healing and decrease postoperative inflammation ${ }^{(6)}$. Regarding postoperative pain, Result of the current study agrees with that of Muñoz et al. using PRF in Combination with traditional bone grafts potentially accelerates wound healing and reduces post-surgical pain ${ }^{(16)}$. Similar result was also reported by Al-Hamed et al and Marenzi et al ${ }^{(19,20)}$. On the other hand, results of the current study is in disagreement with Torul et al and Gülşen et al, They found that PRF seem to have no positive effects pain after surgery ${ }^{(18,21)}$.

In our study we use CBCT for assessment the tooth before extraction, the available bone, anatomy of the area and to avoid unpleasant complications, specifically nerve injury. Results of our study show no case with nerve injury. When treatment planning for an immediate implant in the posterior mandible is planned, a CBCT scan taken before tooth extraction can be of value in assessing the available bone and anatomy of the area. This allows the clinician and patient to consider alternative options, such as treating and maintaining the tooth or using a delayed implant protocol, when the site presents a high risk for immediate implant placement ${ }^{(22)}$. Regarding postoperative nerve injury, Result of the current study agrees with that of Lin et al, who conclude that pre-surgical mapping of the inferior alveolar canal and identification of its proximity relative to the lingual concavity in the posterior mandible regions may avoid unpleasant complications, specifically when performing immediate implant procedures ${ }^{(22)}$.

With respect to Graft exposure and/or loss and soft tissue dehiscence, our results show no soft tissue dehiscence or graft exposure. Dehiscence is opening of the surgical wound edges exposing part or all of the implant head and/or graft with surrounding bony tissues ${ }^{(23)}$. A lot of factors aid in absence of dehiscence or graft exposure in the present study; atraumatic extraction, using MPM in the gap around the implant which present several advantages (it is moldable, contains the mineral phase which is the scaffold for bone cells necessary for bone formation prevent micro and macro movement of grafted bone,Fibrin network entraps platelets and leukocytes),success to ensure closure of the socket with PRF membrane with insufficient or excessive tension on the suture. The absence of dehiscence or graft exposure in the present study is compatible with Abbas et al and Marrelli et al, they found The use of PRF could offer several advantages like promoting soft and hard tissue wound healing ${ }^{(24,25)}$.

Implant stability is an indirect indication of osseointegration, it is a measure of the clinical immobility of an implant. In this study Osstell was used to assess implant stability, with results show increase of ISQ values from base line to the 3 months reading. With reference to stability, results of the current study is in agreement with Sultan et al and Elbokle et al, they found that MPM give successful results regarding implant stability ${ }^{(26,27)}$. 
Results of this study concerning assessment of bone density using CBCT has shown that there was statistically significant increase in bone density after three months. As regard to bone density, results of the present study is in accordance with Sultan et al and Elbokle et al , they found that MPM give successful results regarding bone density ${ }^{(26,27)}$. Also in agreement with Cinar et al who found that MPM, could increase new bone formation ${ }^{(28)}$.

In our study there is no statistically significant change in mean marginal bone loss after three months. Regarding marginal bone loss, our result is in agreement with Uppala et al who found, the addition of PRP results in decreased bone loss around the dental implants ${ }^{(29)}$.

The superiority of MPM in the results of implant stability, bone density gain and marginal bone loss can be explained by the fact that MPM is a homogeneous product that contains important elements for bone formation. It contains the mineral phase which is the scaffold for bone cells necessary for bone formation. It also contains the fibrin network which is the extracellular matrix necessary for migration of specific cells in the tissue regeneration or repair. And it also contains growth factors necessary for the stimulation of differentiation or migration of cells ${ }^{(6)}$.

\section{CONCLUSIONS}

Therefore, it can be concluded that, the use of MPM as grafting material around immediate dental implant in mandibular posterior teeth has a great impact in the outcome of the grafting surgery because MPM is highly concentrated in platelets and fibrin mixed with the mineral phase of bone graft forming a homogeneous single component, which is compact and stable ,containing the graft, and the growth factors promoting healing. This stability of the graft, opened a new age of the use of particulate bone grafts.

\section{REFERENCES}

1. Rajput R, Chouhan Z, Sindhu M, Sundararajan S, Chouhan RR. A brief chronological review of dental implant history. International Dental Journal of Students Research. 2016; 4:105-7.

2. Motamedian SR, Khojaste M, Khojasteh A. Success rate of implants placed in autogenous bone blocks versus allogenic bone blocks: A systematic literature review. Annals of maxillofacial surgery. 2016; 6:78-90.

3. Amine K, Gharibi A, Hsaine A, Kissa J. Effect of Bone Regeneration with Mineralized Plasmatic Matrix for Implant Placement in Aesthetic Zone. Case Reports in Dentistry 2017; 52:70-88.

4. Wang J, Zhu Y, Wang M, Liu D, Chen X, Zhu X, Yang X, Zhang K, Fan Y, Zhang X. Fabrication and preliminary biological evaluation of a highly porous biphasic calcium phosphate scaffold with nano-hydroxyapatite surface coating. Ceramics International. 2018; 44:1304-11.

5. Di Santo MC, Alaimo A, Acebedo SL, Spagnuolo C, Pozner R, Pérez OE. Biological responses induced by high molecular weight chitosan administrated jointly with Platelet-derived Growth Factors in different mammalian cell lines. International Journal of Biological Macromolecules. 2020; 158:953-67.

6. Nadon F, Chaput B, Perisse J, De Berail A, Lauwers F, Lopez R. Interest of mineralized plasmatic matrix in secondary autogenous bone graft for the treatment of alveolar clefts. Journal of Craniofacial Surgery 2015; 26:2148-151.

7. HEALY, Brendan; FREEDMAN, Andrew. ABC of wound healing: infections. BMJ: British Medical Journal, 2006; 332: 838-41.

8. Abu-Mostafa NA. The effects of primary and secondary wound closure following surgical extraction of lower third molars on post-operative morbidity: A prospective randomized clinical trial. J Dent Oral Hyg. 2015; 7:168-74.

9. Chiarotto A, Maxwell LJ, Ostelo RW, Boers M, Tugwell $\mathrm{P}$, Terwee CB. Measurement properties of visual analogue scale, numeric rating scale, and pain severity subscale of the brief pain inventory in patients with low back pain: a systematic review. The Journal of Pain. 2019; 20:245-63.

10. Sennerby L. Resonance frequency analysis for implant stability measurements. A review. Integration Diagn Update. $2015 ; 1: 1-11$.

11. Silva IMCC, Freitas DQ, Ambrosano GMB, Bóscolo FN, Almeida SM. Bone density: comparative evaluation of Hounsfield units in multislice and cone-beam computed tomography, Braz Oral Res., 2012; 26: 550-6. 
12. Kumar V, Sagheb K, KämmererP. Retrospective clinical study of marginalbone level changes with two different Screw-Implant types: comparison between tissue level and bone level implant. J Maxillofac Oral Surg 2014; 13: 259- 66.

13. Marenzi G, Riccitiello F, Tia M, di Lauro A, Sammartino G. Influence of leukocyte-and platelet-rich fibrin (L-PRF) in the healing of simple postextraction sockets: a splitmouth study. BioMed research international. 2015; 2015.

14. Medikeri RS, Meharwade V, Wate PM, Lele SV. Effect of PRF and Allograft Use on Immediate Implants at Extraction Sockets with Periapical Infection-Clinical and Cone Beam CT Findings - . The Bulletin of Tokyo Dental College. 2018; 59:97-109.

15. Hoaglin DR, Lines GK. Prevention of localized osteitis in mandibular third-molar sites using platelet-rich fibrin. International journal of dentistry. 2013; 2013.

16. Muñoz F, Jiménez C, Espinoza D, Vervelle A, Beugnet J, Haidar Z. Use of leukocyte and platelet-rich fibrin (LPRF) in periodontally accelerated osteogenic orthodontics (PAOO): Clinical effects on edema and pain. Journal of clinical and experimental dentistry. 2016; 8:e119-24.

17. Ozgul O, Senses F, Er N, Tekin U, Tuz HH, Alkan A, Kocyigit ID, Atil F. Efficacy of platelet rich fibrin in the reduction of the pain and swelling after impacted third molar surgery: Randomized multicenter split-mouth clinical trial. Head \& face medicine. 2015; 11:1-5.

18. Torul D, Omezli MM, Kahveci K. Evaluation of the effects of concentrated growth factors or advanced platelet richfibrin on postoperative pain, edema, and trismus following lower third molar removal: A randomized controlled clinical trial. Journal of Stomatology, Oral and Maxillofacial Surgery. 2020.

19. Al-Hamed FS, Tawfik MA, Abdelfadil E. Clinical effects of platelet-rich fibrin (PRF) following surgical extraction of lower third molar. The Saudi Journal for Dental Research. 2017; 8:19-25.

20. Marenzi G, Riccitiello F, Tia M, di Lauro A, Sammartino G. Influence of leukocyte-and platelet-rich fibrin (L-PRF) in the healing of simple postextraction sockets: a split-mouth study. BioMed research international. 2015; 2015:369273.
21. Gülşen U, Şentürk MF. Effect of platelet rich fibrin on edema and pain following third molar surgery: a split mouth control study. BMC oral health. 2017; 17:79-84.

22. Lin MH, Mau LP, Cochran DL, Shieh YS, Huang PH, Huang RY. Risk assessment of inferior alveolar nerve injury for immediate implant placement in the posterior mandible: a virtual implant placement study. Journal of dentistry. 2014; 42:263-70.

23. Samir E, Hicham S, Keltoum EO, Zouheir I. Management of post-extractional alveolar socket with mineralized plasmatic matrix before implant placement: a case report 2017; 4:220-7.

24. Abbas AM, Bede SY, Alnumay SH. Evaluation of the effectiveness of using platelet rich fibrin (PRF) as a sole grafting material and membrane in augmentation of dehiscence and fenestration defects encountered during dental implant surgery. Journal of Baghdad College of Dentistry. 2019; 31:44-51.

25. Marrelli M, Tatullo M. Influence of PRF in the healing of bone and gingival tissues. Clinical and histological evaluations. Eur Rev Med Pharmacol Sci. 2013; 17:1958-62.

26. Sultan OI, Chehata IM, Hossam AM. Implant Stability Parameters \& Bone Density Values of Different Graft Materials with Immediately Placed Dental Implants. Egyptian Dental Journal. 2018; 64:3135-48.

27. Elbokle NN, Sultan OI, Chehata IM, Hossam AM. Effect Of Bone Regeneration With Platelets Rich Fibrin Versus Mineralized Plasmatic Matrix For Immediate Implant Placement. Egyptian Dental Journal. 2017; 63:3057-67.

28. Cinar IC, Gultekin BA, Saglanmak A, Yalcin S, Olgac V, Mijiritsky E. Histologic, Histomorphometric, and Clinical Analysis of the Effects of Growth Factors in a Fibrin Network Used in Maxillary Sinus Augmentation. International Journal of Environmental Research and Public Health. 2020; 17:1918.

29. Uppala S, Parihar AS, Modipalle V, Manual L, Oommen VM, Karadiguddi P, Gupta P. Crestal bone loss around dental implants after implantation of Tricalcium phosphate and Platelet-Rich Plasma: A comparative study. Journal of Family Medicine and Primary Care. 2020; 9:229-34. 